\title{
Optical Coherence Tomography and Coronary Plaque Characterization
}

\author{
Tsunenari Soeda, ${ }^{1}$ Shiro Uemura, ${ }^{2}$ Yoshihiko Saito,${ }^{2}$ Kyoichi Mizuno, ${ }^{3}$ Ik-Kyung Jang ${ }^{1}$
}

\section{Introduction}

Since the first percutaneous balloon angioplasty was performed by Andreas Gruntzig in 1977,, 2) percutaneous coronary intervention (PCI) has become the primary mode of revascularization for coronary artery disease (CAD). The invention of bare metal stents and the introduction of drug eluting stents significantly improved the outcomes. ${ }^{3-9)}$ Imaging modalities such as intravascular ultrasound (IVUS) and intravascular angioscopy have also been developed, which provide us crucial information for understanding of CAD and planning PCI. In recent decades, intravascular optical coherence tomography (OCT) has been extensively utilized both in research and in the setting of PCI. We will briefly introduce OCT and focus on plaque characterization utilizing OCT.

\section{Introduction of OCT}

OCT was developed by two different groups in the late 1980s. ${ }^{10,11)}$ Tanno, et al applied for the Japanese Patent for a concept of OCT in 1990. ${ }^{12}$ ) The first OCT was demonstrated by Huang, et al at the Massachusetts Institute of Technology in the USA. ${ }^{13)}$ They reported the possibilities of in vitro imaging at the peripapillary area of the retina and in the coronary artery. In 1995, Brezinski, et al reported that they succeeded in visualizing an intimal fibrous cap overlying lipid contents using an intracoronary OCT fiber. ${ }^{14)}$ The same OCT fiber was also demonstrated in vivo endoscopic imaging in rabbit gastrointestinal and respiratory tracts. ${ }^{15)}$ In 1998, Fujimoto, et al successfully imaged the rabbit aorta in vivo with a resolution of $10 \mu \mathrm{m} .{ }^{16)}$ Then the cardiac OCT research group was established at the Massachusetts General Hospital (MGH), to create objective OCT image criteria for atherosclerotic plaque characterization. Yabushita, et al evaluated diseased atherosclerotic segments from coronary autopsies and they established the criteria, which demonstrated high sensi-

${ }^{1}$ Cardiology Division, Massachusetts General Hospital, Harvard Medical School, Boston, Massachusetts, USA (GRB 800, 55 Fruit Street, Boston, MA, USA), ${ }^{2}$ The First Department of Medicine, Nara Medical University, Kashihara, Japan, ${ }^{3}$ Department of Cardiovascular Medicine, Nippon Medical School, Tokyo, Japan

doi: 10.7793 jcoron. 19.033 tivity and specificity for atherosclerotic coronary plaques. ${ }^{17}$ Based on this autopsy data and successful visualization of coronary arteries in living porcine, ${ }^{18)}$ the first-in-man study was performed in 2000 and the feasibility of intravascular OCT for visualizing the characteristics of atherosclerotic coronary plaques in humans was tested. ${ }^{19)}$ In this study, intracoronary OCT safely identified most architectural features. This was the crucial first step for human research with OCT.

As research progressed, clinical implementation, particularly in PCI, was important and commercial development was necessary to increase the use of OCT. LightLab Imaging, Inc (Westford, MA, USA) was established in 1998 and was acquired by Goodman Ltd (Nagoya, JAPAN) in 2002. LightLab Imaging, Inc introduced the first generation of OCT in Europe with the M2 imaging system which had the capability of imaging 15 frames per second and 200 axial scans per frame with an occlusion balloon system to remove blood flow. The M3 system was introduced in Japan at 2007 with improvement of imaging speeds (20 frames/s and 240 axial scans/frame). The catheter system was later refined to accommodate Fourier-domain OCT imaging. In 2009, the C7XR ${ }^{\mathrm{TM}}$ system, which could acquire 100 frames/s (500axial scans/frame) without using an occlusion balloon system, was introduced at the US and Europe. In 2011, LightLab Imaging, Inc was sold to St. Jude Medical (St. Paul, MN, USA). The next generation OCT system (ILUMIEN ${ }^{\mathrm{TM}}$ OCT Intravascular Imaging Systems) had an additional function of wireless fractional flow reserve measurement. In 2012, ILUMIEN ${ }^{\mathrm{TM}}$ Optis $^{\mathrm{TM}}$ OCT Intravascular Imaging System with a faster pull-back speed $(36 \mathrm{~mm} / \mathrm{s})$, longer pull-back length $(75 \mathrm{~mm})$ and 3D image optimization was approved in Japan. These improvements in imaging speeds and system performance allowed wide spread use.

The principle of OCT is analogous to IVUS using near-infrared light as the imaging tool instead of acoustic waves. ${ }^{10,11,13)}$ Nearinfrared light is focused into the tissue, and the echo time delay of the light reflected from the internal microstructure at different depths is measured by the detector. Image information is obtained by performing repeated axial measurements at different transverse positions, while the optical beam is scanning across the tissue. The resulting data is reconstructed to a two-dimensional map of the backscattering or reflectance from internal architectural mor- 
phology and cellular structures in the tissue (Fig. 1A). ${ }^{20)}$ The first generation OCT systems utilized a near-infrared light source and determined tissue depth by moving the distance to the reference mirror. This system has become recognized as time-domain OCT. In ophthalmology, this system is widely established for research and clinical use. But, as an intravascular tool, this OCT system has not been adopted for routine practice because of some limitations. As blood is nearly opaque to near-infrared light, the first generation OCT required the occlusion of coronary flow and displacement of blood with contrast or saline for more than $30 \mathrm{sec}-$ onds in vivo. Also, this system could only get a few images per second from isolated locations within the coronary arteries. To improve these limitations, the second generation OCT was designed using wavelength-swept laser as the light source and fixed mirror as a reference (Fig. 1B). Wavelength-swept laser is a tunable light source with a sweep range of $1,250-1,350 \mathrm{~nm}^{21,22)}$ The detector calculates optical wavelength and time to reconstruct tissue images. This method enables OCT to increase frames taken per second by more than 10-fold and allows greater penetration depth. This system is called as frequency-domain OCT (FDOCT), Fourier-domain OCT, swept-source OCT (SS-OCT), or optical frequency-domain imaging (OFDI).

\section{Clinical application}

\section{Plaque characterization}

As OCT has a high temporal resolution of $10 \mu \mathrm{m}$, it allows us to identify the normal coronary arterial wall and various atherosclerotic plaque morphologies. ${ }^{17,19,23-28)}$ Normal arterial wall is characterized by a three-layered structure, comprising an intima visualized as a highly backscattering or signal-rich layer, a media observed as a low backscattering or signal poor region, and a heterogeneous and highly backscattering adventitia (Fig. 2A). The internal elastic membrane is defined as signal-rich band between the intima and media, and the external elastic membrane is defined as the signal-rich band between the media and the adventitia. ${ }^{19)}$

An atherosclerotic plaque is defined as a focal thickening of intimal layer or destructions of a layered structure of the coronary wall. Fibrous plaque is typically defined as a high backscattering and a relatively homogeneous signal. Sometimes, the adventitia is visualized in fibrous plaques (Fig. 2B). ${ }^{17,19)}$ Due to limited penetration depth, some fibrous tissues are not accurately detected. Lipid core is defined as a signal-poor region with poorly delineated borders and covered with an overlying signal-rich band that is recognized as fibrous cap. ${ }^{17,}$ 19) Lipid-rich plaque is defined as a plaque with more than one quadrant of lipid core in a cross-sectional image (Fig. 2C). ${ }^{25)}$ Fibrocalcific plaque is identified by the presence of fibrous tissue overlying calcific contents. Calcification is defined as a signal-poor or heterogeneous region with a sharply delineated border (Fig. 2D). $\left.{ }^{17}, 19\right)$

In the initial study from our group, which validated OCT findings with histology, the sensitivity and specificity for the detection of fibrocalcific plaque was 95 to $96 \%$ and $97 \%$, lipid-rich plaque was 90 to $94 \%$ and 90 to $92 \%$, respectively. ${ }^{17)}$ The sensitivity of fibrous plaque was relatively low (71 to 79\%), but the specificity remained high (97 to $98 \%$ ) and the negative predictive value was 91 to $93 \%$. Adapted to these criteria, further observational study were performed in patients with CAD including stable angina pectoris (SAP), ST elevated myocardial infarction (STEMI), and non-ST elevated acute coronary syndrome (NSTE-ACS). Lipid-rich plaque, which is defined as plaque with large lipid core occupying more than 90 degrees in a cross-sectional image, was more frequently observed in STEMI and NSTE-ACS compared with SAP. ${ }^{23)}$

\section{Vulnerable plaque}

Vulnerable plaque is prone to rupture and coronary thrombosis resulting in ACS and sudden cardiac death. The morphological characteristics for ruptured plaques ${ }^{29-33)}$ are observed in the lesions with thin-cap fibroatheroma (TCFA), which has a thin fibrous cap ${ }^{34)}$ overlying large eccentric necrotic core, ${ }^{32)}$ macrophage accumulation, ${ }^{35)}$ neovascularization, ${ }^{36)}$ and spotty calcification. Although 60 to $70 \%$ of ACS result from the rupture of a TCFA, the remaining causes of ACS are mostly related to intimal erosion ${ }^{33)}$ or calcific nodules ${ }^{32}$ ) with subsequent local thrombus formation. ${ }^{29)}$

\section{2-a. Thin capped fibroatheroma (TCFA)}

TCFA is pathologically defined as a necrotic core with an overlying fibrous cap measuring $<65 \mu \mathrm{m}$ with numerous macrophages. ${ }^{32,34,35)}$ OCT is the only intravascular modality that can measure fibrous cap thickness. By definition, OCT derived TCFA is characterized as a plaque with $>90$ degrees of lipid core and $<65 \mu \mathrm{m}$ of fibrous cap thickness (Fig. 3A). ${ }^{23)}$ Using this criteria, TCFA was observed in $72 \%$ of culprit lesions of STEMI and $50 \%$ of NSTE-ACS patients as compared to $20 \%$ of SAP $\left(\mathrm{P}=0.012\right.$, respectively). ${ }^{23)}$ The thickness of fibrous cap at the culprit lesion was thinner in STEMI and NSTE-ACS than in SAP patients $(47.0 \mu \mathrm{m}, 53.8 \mu \mathrm{m}$, and $102.6 \mu \mathrm{m}$, respectively, $\mathrm{P}=0.034)$. In a comparison study of OCT and coronary angioscopy, vulnerable yellow plaques identified by coronary angioscopy are correlated with a thinner fibrous cap. ${ }^{37,38)}$ As OCT has limitations of penetration depth, it can not detect positive remodeling, but the combination of OCT and IVUS proved positive remodeling was more commonly associated with lipid-rich plaque $(100 \%, 60 \%$, and $47.4 \%, \mathrm{P}=0.01)$, a thin fibrous cap (median $40.2 \mu \mathrm{m}, 51.6 \mu \mathrm{m}$, and $87 \mu \mathrm{m}, \mathrm{P}=0.003)$ and the presence of TCFA $(80 \%, 38.5 \% \text {, and } 5.6 \%, \mathrm{P}<0.001)^{39)}$ compared with absent or negative remodeling. The patients with TCFA have 


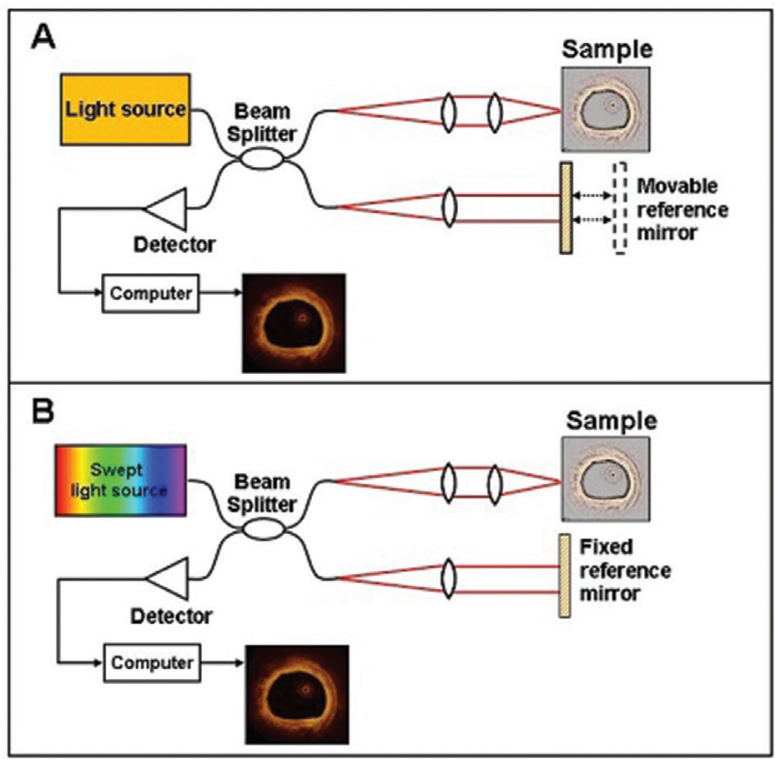

Fig. 1 Schema of time-domain OCT (A) and frequency-domain OCT (B).

higher inflammatory markers, such as high sensitivity-C reactive protein (hs-CRP), IL-18, ${ }^{40)}$ and Pentraxin $3 .{ }^{41)}$ These findings may suggest that TCFA is more frequently seen in patients with unstable condition.

\section{2-b. Macrophage accumulation}

Increased infiltration of macrophages into the plaque cap is the most common sign of high risk coronary plaques. ${ }^{34,42)}$ Macrophage accumulation is seen as bright spots with high OCT backscattering attenuation (Fig. 3B). ${ }^{43,44)}$ Our group has reported that OCT has an ability to detect plaques with greater than $10 \%$ macrophage density within the fibrous cap, which was quantified as CD68 positive cell in an autopsy study. ${ }^{44)}$ Although it was a small study, ACS patients have greater macrophage accumulations in the culprit lesions compared with SAP. ${ }^{43)}$ Our group also reported that greater macrophage accumulation is associated with peripheral white blood cell count and inversely correlated with fibrous cap thickness of fibroatheroma. ${ }^{45}$

\section{2-c. Microchannel}

Neovascularization is pathologically reported as an important process in the progression of atherosclerosis ${ }^{42)}$ and has also been recognized as one of the features of vulnerable plaque. ${ }^{36)}$ OCT has an ability to detect a neovascularization as a microchannel, defined as small black hole or a tubular structure without a connection to the vessel lumen thickness (Fig. 3C). ${ }^{46)}$ In a postmortem study, adventitial microvessels are more frequently seen in symptomatic atherosclerosis. ${ }^{42)}$ Kitabata, et al have reported that plaque with microchannels is correlated with a thinner fibrous cap, a high frequency of positive remodeling and TCFA using OCT and IVUS. ${ }^{46)}$ They also reported that an increased number
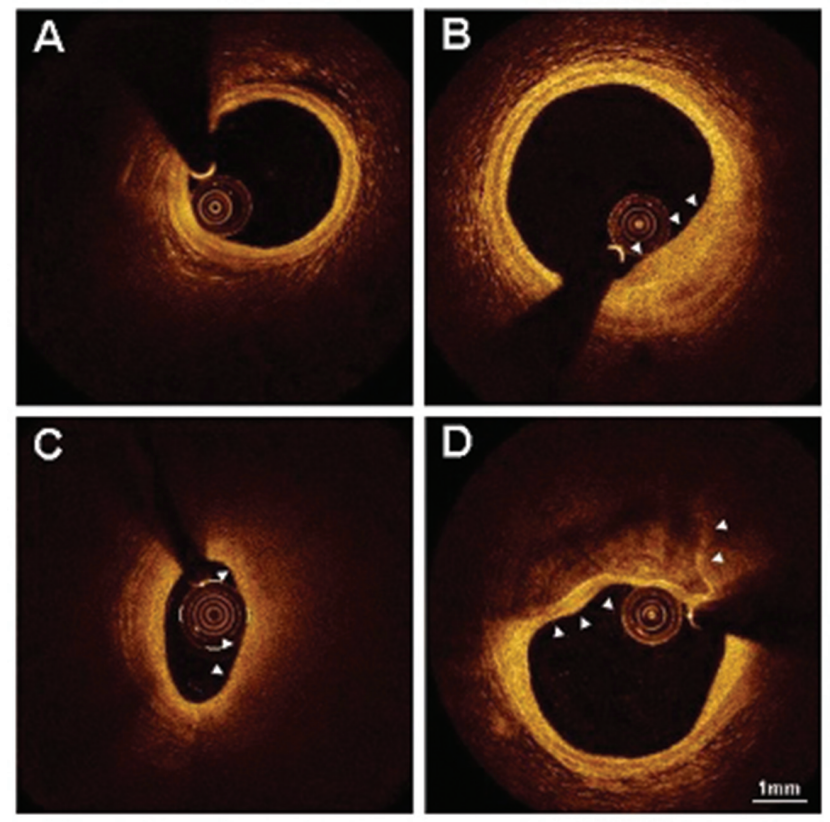

Fig. 2 Cross-sectional optical coherence tomography images of atherosclerotic plaques.

A: Normal coronary arterial wall.

B: Fibrous plaque; Fibrous plaque is typically defined as a high backscattering and a relatively homogeneous signal (arrowheads).

C: Lipid-rich plaque; Lipid-rich plaque is defined as a plaque with more than one quadrant of lipid core in cross-sectional image (arrowheads).

D: Fibrocalcific plaque; Calcification is defined as a signal-poor or heterogeneous region with a sharply delineated border (arrowheads).
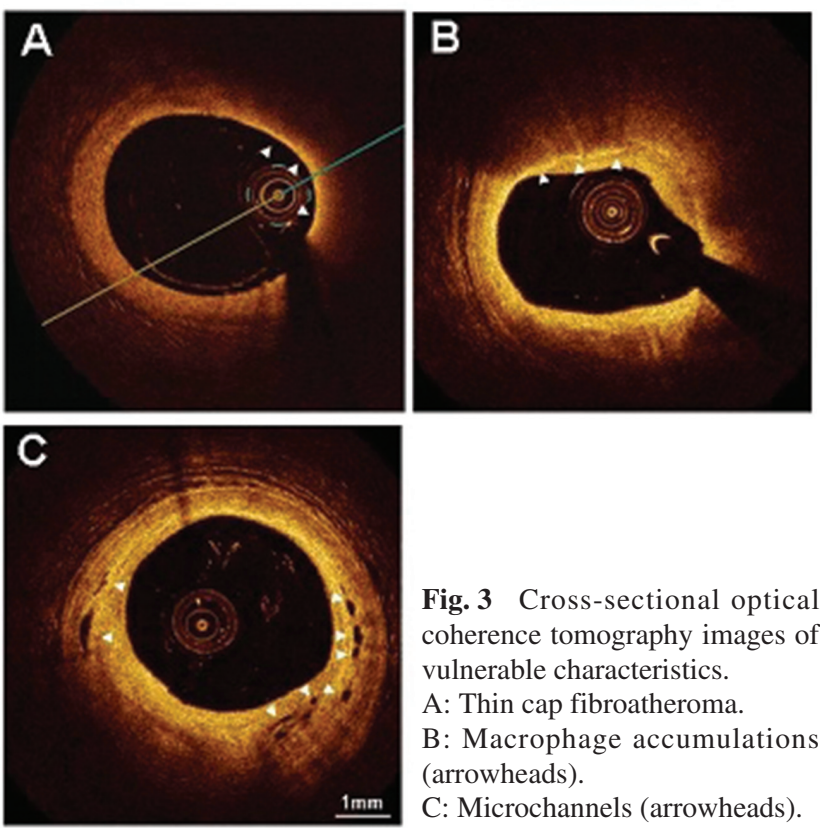

Fig. 3 Cross-sectional optical coherence tomography images of vulnerable characteristics.

A: Thin cap fibroatheroma. B: Macrophage accumulations (arrowheads).

C: Microchannels (arrowheads).

of microchannels are associated with higher hs-CRP. In patients with unstable angina pectoris, culprit lesions with microchannels had significantly higher incidence of TCFA, thinner fibrous cap, greater lipid arc and longer lipid core length compared to culprit 
lesions without microchannels. ${ }^{47)}$

\section{Plaque progression}

Using OCT detected vulnerable features, Uemura, et al have evaluated the morphological characteristics of non-significant coronary plaques which developed rapid progression of coronary stenosis in patients with CAD. Compared to plaques without progression, plaques with progression showed a significant higher incidence of lipid pools ( $100 \%$ and $60.7 \%, \mathrm{P}=0.02)$, microchannel (76.9\% and 14.3\%, P<0.01), TCFA (76.9\% and $14.3 \%, \mathrm{P}<0.01)$, and macrophage accumulations $(61.5 \%$ and $\left.14.3 \%, \mathrm{P}<0.01) .{ }^{48}\right)$ And TCFA and microchannel showed high potential to predict rapid progression of non-significant coronary plaques.

\section{Acute coronary syndrome}

\section{4a. Plaque rupture}

Extensive study has focused on the fibrous cap of the lipid core because the majority of ACS is caused by rupture of the fibrous cap. ${ }^{49)}$ When rupture of the fibrous cap occurs, the thrombogenic contents within the lipid core flow into the blood and results in luminal thrombosis. ${ }^{50)}$ In an autopsy study of sudden coronary death, 55 to $60 \%$ subjects have plaque rupture as the etiology of ACS. ${ }^{35,51,52)}$ Plaque rupture is now pathologically defined as a structural defect -a gap- in the fibrous cap that separates the lipidrich necrotic core of a plaque from the lumen of the artery. ${ }^{30)}$

As previously mentioned OCT can detect thin fibrous cap, OCT can also detect the ruptured plaque in ACS patients (Fig. 4A). In an OCT study, 73\% of plaque rupture was found in the patients with acute myocardial infarction. ${ }^{25)}$ Yonetsu, et al have analyzed 103 culprit lesions from patients with ACS before PCI using OCT. They reported that the median thinnest cap thickness was $54 \mu \mathrm{m}$ (IQR: 50-60 $\mu \mathrm{m}$ ) in the ruptured plaques and the thinnest cap thickness was less than $80 \mu \mathrm{m}$ in $95 \%$ of ruptured plaques. ${ }^{53)}$ Tanaka, et al have analyzed 43 consecutive ACS patients with a ruptured plaque in the culprit lesion using OCT. The subjects were divided into a rest and an exertion group based on their activities at the onset of ACS. The median thickness of the disrupted fibrous cap in the restonset group is significantly thinner $(50 \mu \mathrm{m}$, interquartile median $15 \mu \mathrm{m}$ ) compared with the exertion group (90 $\mu \mathrm{m}$, interquartile median $65 \mu \mathrm{m})(\mathrm{P}<0.01) .{ }^{54)}$ Thinner fibrous cap is one of the important signs for plaque rupture, but some of plaque ruptures may occur in thick fibrous caps with exertion. On the other hand, silent plaque rupture is also reported as a common phenomenon in autopsy studies. ${ }^{55,56)}$ Silent plaque ruptures were seen $35.3 \%$ of ACS patients and $12.6 \%$ of non-ACS patients. ${ }^{57)}$ Silent plaque ruptures are more frequently seen in patients with chronic kidney disease. ${ }^{58)}$ Although acute events occur from plaque rupture with mural thrombosis, repeat silent plaque rupture advances coronary stenosis by stages and leads to severe stenosis. ${ }^{56,59,60)}$

\section{4b. Erosion}

Plaque erosion is seen in 30 to $35 \%$ of autopsy cases of sudden coronary death. ${ }^{60)}$ Plaque erosion is pathologically identified as the thrombosed arterial lesion without plaque rupture. ${ }^{35)}$ Typically, the endothelium is lacking at the erosion site, and the exposed intima consists predominantly of vascular smooth muscle cells and proteoglycans. The underlying plaque morphology shows the presence of pathological intimal thickening or a fibroatheroma with an intact media. Generally, eroded lesions have few inflammatory infiltrations. A clinical OCT study with AMI showed $23 \%$ of plaque erosion. ${ }^{25)}$ In this study, erosion was defined that "fibrous cap erosion" was characterized by loss of the endothelial lining with lacerations of the superficial intimal layers and without trans-cap ruptures.

\section{4c. Calcified nodule}

Calcified nodule is pathologically defined as a plaque which shows presence of breaks in the calcified plate and interspersed fibrin with disrupted surface fibrous cap and an overlying thrombus. ${ }^{32,35)}$ Approximately 2 to $7 \%$ of coronary thrombosis in STEMI was caused by calcified nodules. ${ }^{32,61)}$

4d. New OCT definition for plaque rupture, erosion, and calcified nodule

Due to limitations of OCT resolution and penetration depth, we cannot visualize the endothelial layer and massive thrombus prevents us from evaluating the condition of the fibrous cap. We have established an OCT criteria for plaque rupture, erosion and calcified nodules, collaborating with pathologists (Fig. 5). ${ }^{62)}$ The culprit lesions are classified as plaque rupture, erosion (OCT-erosion), calcified nodule (OCT-calcified nodule; OCT-CN), and others, which include tight stenosis, dissection, hematoma, coronary spasm, and fissure. As the OCT evaluation for erosion is different from the pathological definition, we used the term of "OCT-erosion." OCT-erosion is a plaque without fibrous cap disruption and is categorized by the presence of thrombus. If the lesion has overlying thrombus, it is identified as "definite OCT-erosion" (Fig. 4B). But, as there is no thrombus or thrombus masking underlying plaque in some cases, we established another category "probable OCTerosion." Probable OCT-erosion is defined by: 1) luminal surface irregularity at the culprit lesion without overlying thrombus; or 2) attenuation of underlying plaque by thrombus without superficial lipid or calcification immediately proximal or distal to the site of thrombus. OCT-CN was defined when fibrous cap disruption was detected over a calcified plaque characterized by protruding calcification, superficial calcium, and the presence of substantive calcium proximal and/or distal to the lesion (Fig. 4C).

Using this definition, we evaluated 126 culprit lesions of ACS. 

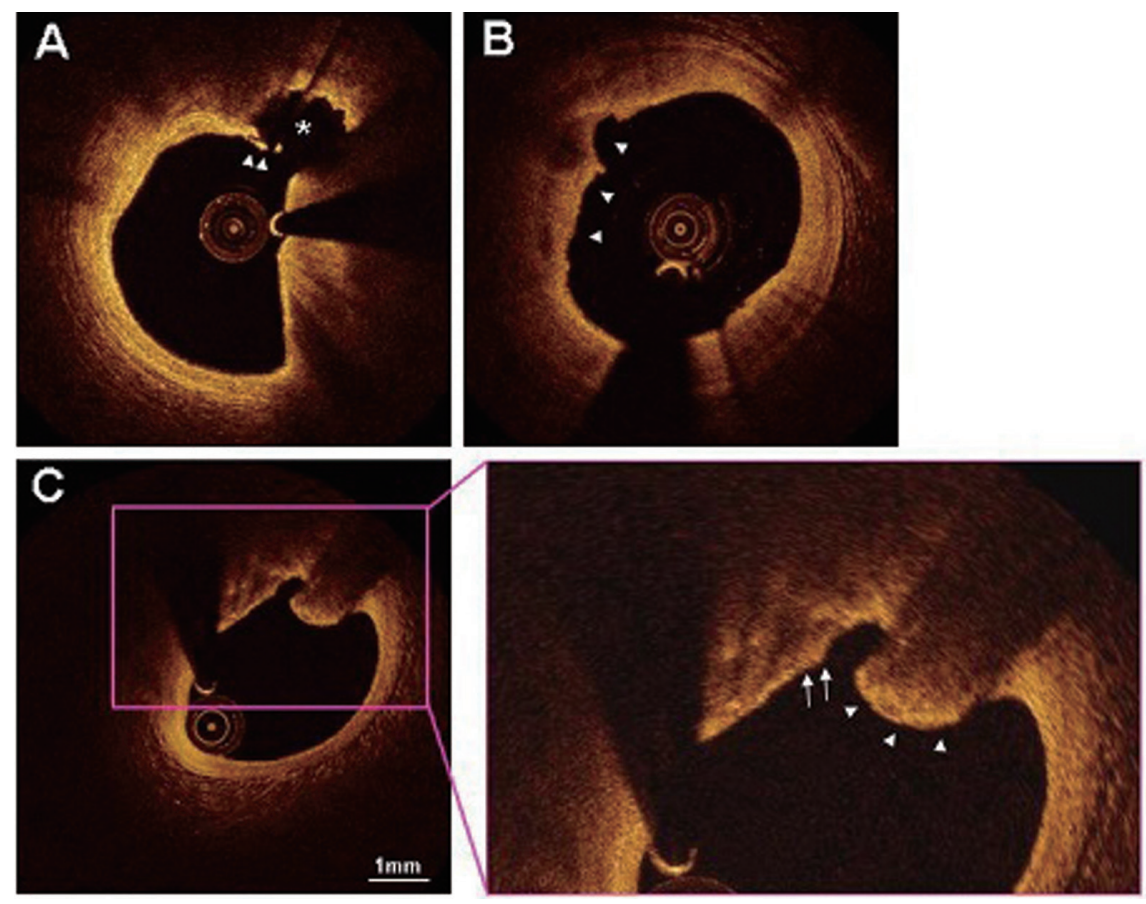

Fig. 4 Representative OCT images of acute coronary syndrome.

A: Plaque rupture; Cross-sectional image shows rupture of fibrous cap (arrowheads) and a cavity (*) formation in the lipid core.

B: Definite OCT-erosion; Arrowheads show thrombus without disruption of fibrous cap.

C: OCT calcified nodule; Cross-sectional image shows fibrous cap disruption over the calcified plaque (white arrow) and protrusion calcium nodule with an overlying fibrin (arrowheads).

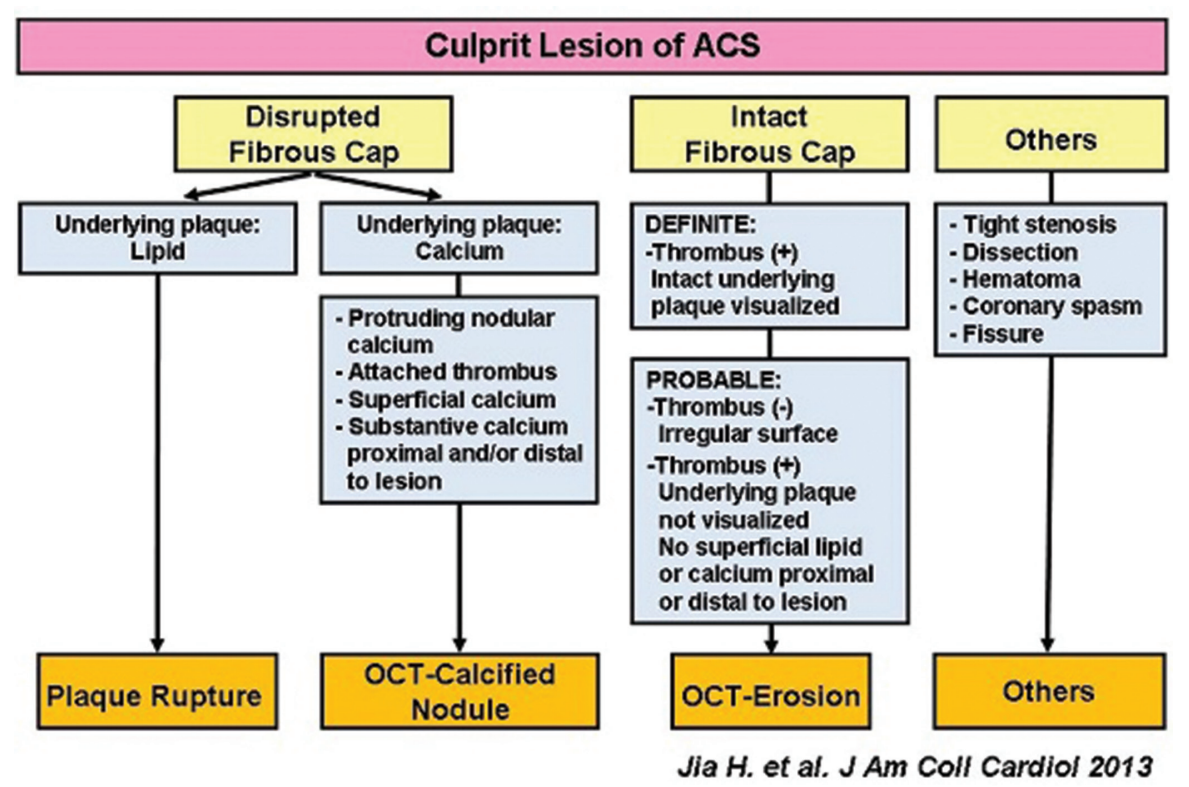

Fig. 5 Plaque classification algorithm for acute coronary syndrome by OCT.

This image is cited from reference 62 (license number: 3240381416890 ).

We observed 55 plaque ruptures (43.7\%), 39 OCT-erosions (31.0\%), and 10 OCT-CNs (7.9\%). From 39 OCT-erosions, 23 lesions $(18.3 \%)$ were definite OCT-erosions and $16(12.7 \%)$ were probable OCT-erosions. Compared with plaque rupture lesions, OCT-erosion lesions were less frequent than lipid plaques (44.6\% vs $100 \%, \mathrm{P}<0.001)$. And lipid-rich plaque detected underneath OCT-erosion has thicker fibrous cap, smaller lipid angle, and shorter lipid length than lipid-rich plaque with plaque rupture. ${ }^{62)}$

\section{4e. Other causes of acute coronary syndrome}

Spontaneous coronary artery dissection is clinically rare, but frequently described as a cause of ACS. ${ }^{63,64)}$ Dissection of the coronary artery separates the arterial wall into the different layers to create a false lumen. The dissection can occur between the intima and media or between the media and adventitia. The false lumen is filled with hemorrhage or hematoma and the true lumen of the coronary artery is suppressed. Sometimes this phenomenon is the result of a non-occlusive or occlusive obstruction of 


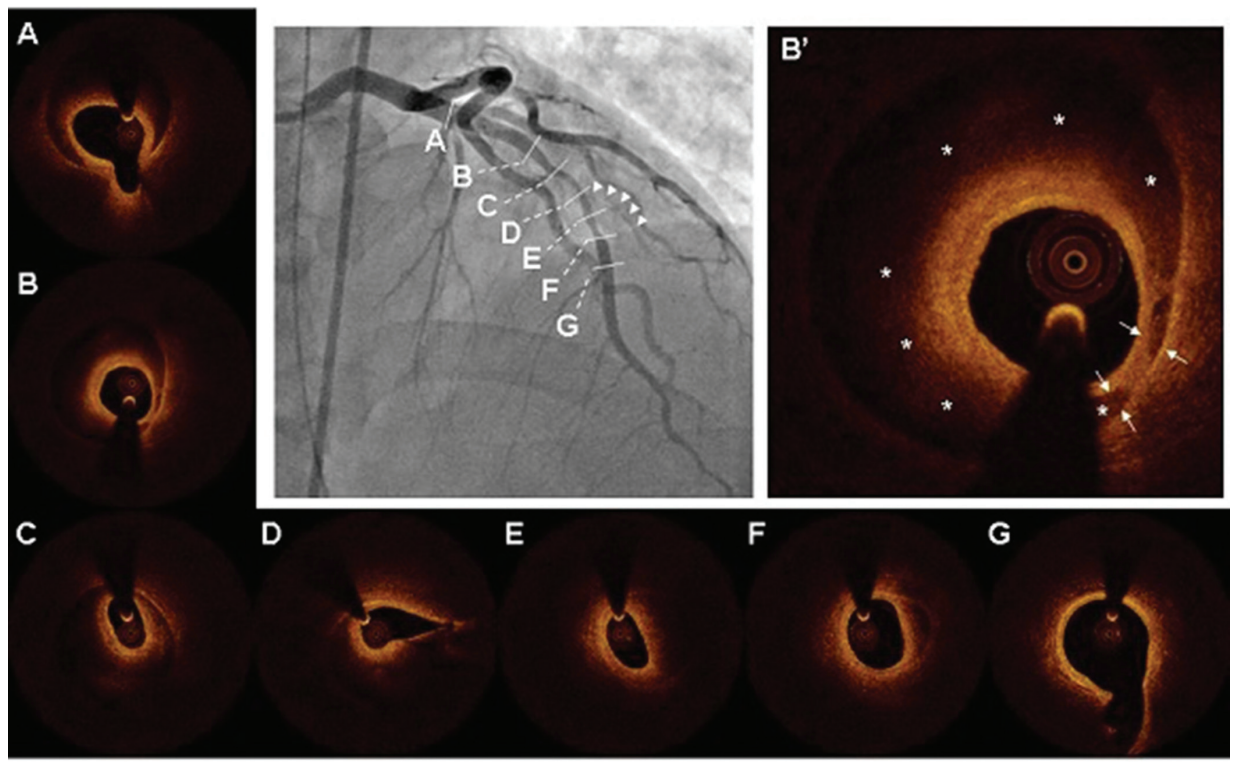

Fig. 6 A case of spontaneous coronary dissection.

A 42-year-old female presented nonST-elevated myocardial infarction. Coronary angiography showed moderate stenosis in the mid LAD, and obstructed flow in the second diagonal branch (white arrowhead). But dissection could not be differentiated.

Coronary dissection was seen from first major septal branch (A) to mid LAD (F) in the cross-sectional OCT images. B' is magnified an OCT image of $B$. The dissection is observed between the media and adventitia (white arrows). The guidewire and OCT catheter were located in the true lumen. The false lumen is filled with hematoma (*). The entry and reentry points were not clearly identified in this case.

the blood flow. ${ }^{64)}$ OCT is one of the powerful tools to detect coronary dissection. ${ }^{65-67)}$ When PCI is planned for coronary dissection, OCT provides us with a precise entry point, dissected length, differentiation of the true from the false lumen, and visualization of the origin of the major side branches (Fig. 6).

Coronary spasm is one of the causes of ACS. This is infrequent in ACS cases, ${ }^{62)}$ but it is often seen as the cause of atypical angina. From an OCT study, coronary spasms occur more frequently at the fibrous lesion compared with the lipid-rich lesion or calcified lesion. ${ }^{68)}$ There is no typical OCT finding when spasm has not occurred. But, in vivo OCT study has reported spasm as medial contraction which occurs even in an asymptomatic state and facilitates the intima in patients with vasospastic angina. ${ }^{69)}$

\section{MGH-OCT registry}

As described before, OCT has been used in research for in vivo vascular biology. However, we still do not have enough data on long term clinical outcomes and prognosis of these OCT findings. To answer these questions, the MGH-OCT registry was launched in 2010. The aims of this project are to 1) identify plaque characteristics in OCT that are associated with adverse cardiac events including ACS in the future, and 2) to identify characteristics of stented arteries that are associated with in-stent restenosis and stent thrombosis. Currently, 21 sites from 6 countries (Australia 3, China 2, Japan 5, Korea 5, Singapore 1, and United States 5) participate in this Registry. We have more than 2,000 cases with a final target of 3,000 cases including clinical follow-up of 5 years. We believe that this registry may be able to answer a lot of unsolved questions in OCT.

\section{Conclusion}

OCT helps us to understand in vivo pathobiology of CAD, especially features consistent with vulnerable plaque. However, there remains the question; what is the clinical significance of these findings.

\section{Acknowledgments}

The authors thank all the investigators, all supporting staff, and all the sites of MGH OCT Registry for their contributions. We also thank all the fellows, Iris A McNulty, and James Chan for exceptional work in the core laboratory, and Russell Joye for his editorial knowledge.

\section{Disclosure}

Dr. IK Jang received a research grant and consulting fee from LightLab Imaging/St. Jude Medical.

\section{References}

1) Gruntzig A: Transluminal dilatation of coronary-artery stenosis. Lancet 1978; 1: 263

2) Grüntzig A, Myler R, Hanna E, et al: Circulation 1977; 56: 84

3) Sigwart U, Puel J, Mirkovitch V, et al: Intravascular stents to prevent occlusion and restenosis after transluminal angioplasty. N Engl J Med 1987; 316: 701-706

4) Roubin GS, Cannon AD, Agrawal SK, et al: Intracoronary stenting for acute and threatened closure complicating percutaneous transluminal coronary angioplasty. Circulation 1992; 85: 916-927

5) Carrozza JP, Kuntz RE, Levine MJ, et al: Angiographic and clinical outcome of intracoronary stenting: immediate and long- 
term results from a large single-center experience. J Am Coll Cardiol 1992; 20: 328-337

6) Serruys PW, de Jaegere P, Kiemeneij F, et al: A comparison of balloon-expandable-stent implantation with balloon angioplasty in patients with coronary artery disease. Benestent Study Group. N Engl J Med 1994; 331: 489-495

7) Kimura T, Yokoi H, Nakagawa Y, et al: Three-year follow-up after implantation of metallic coronary-artery stents. N Engl J Med 1996; 334: 561-566

8) Moses JW, Leon MB, Popma JJ, et al: Sirolimus-eluting stents versus standard stents in patients with stenosis in a native coronary artery. N Engl J Med 2003; 349: 1315-1323

9) Colombo A, Drzewiecki J, Banning A, et al: Randomized study to assess the effectiveness of slow- and moderate-release polymerbased paclitaxel-eluting stents for coronary artery lesions. Circulation 2003; 108: 788-794

10) Takada K, Yokohama I, Chida K, et al: New measurement system for fault location in optical waveguide devices based on an interferometric technique. Appl Opt 1987; 26: 1603-1606

11) Youngquist RC, Carr S, Davies DE: Optical coherence-domain reflectometry: a new optical evaluation technique. Opt Lett 1987; 12: 158-160

12) Tanno N, Ichimura $T$, Saeki A: Device for measuring light wave of a reflected image. Japanese Patent 1990; Japanese Patent 2010042:

13) Huang D, Swanson EA, Lin CP, et al: Optical coherence tomography. Science 1991; 254: 1178-1181

14) Brezinski ME, Tearney GJ, Bouma BE, et al: Optical coherence tomography for optical biopsy. Properties and demonstration of vascular pathology. Circulation 1996; 93: 1206-1213

15) Tearney GJ, Brezinski ME, Bouma BE, et al: In vivo endoscopic optical biopsy with optical coherence tomography. Science 1997; 276: 2037-2039

16) Fujimoto JG, Boppart SA, Tearney GJ, et al: High resolution in vivo intra-arterial imaging with optical coherence tomography. Heart 1999; 82: 128-133

17) Yabushita H, Bouma BE, Houser SL, et al: Characterization of human atherosclerosis by optical coherence tomography. Circulation 2002; 106: 1640-1645

18) Tearney GJ, Jang IK, Kang DH, et al: Porcine coronary imaging in vivo by optical coherence tomography. Acta Cardiol 2000; 55: 233-237

19) Jang IK, Bouma BE, Kang DH, et al: Visualization of coronary atherosclerotic plaques in patients using optical coherence tomography: comparison with intravascular ultrasound. J Am Coll Cardiol 2002; 39: 604-609

20) Tearney GJ, Brezinski ME, Bouma BE, et al: In vivo endoscopic optical biopsy with optical coherence tomography. Science 1997; 276: 2037-2039

21) Chinn SR, Swanson EA, Fujimoto JG: Optical coherence tomography using a frequency-tunable optical source. Opt Lett 1997; 22: 340-342

22) Bouma BE, Yun SH, Vakoc BJ, et al: Fourier-domain optical coherence tomography: recent advances toward clinical utility. Curr Opin Biotechnol 2009; 20: 111-118

23) Jang IK, Tearney GJ, MacNeill B, et al: In vivo characterization of coronary atherosclerotic plaque by use of optical coherence tomography. Circulation 2005; 111: 1551-1555

24) Kume T, Akasaka T, Kawamoto T, et al: Assessment of coronary arterial plaque by optical coherence tomography. Am J Cardiol 2006; 97: 1172-1175

25) Kubo T, Imanishi T, Takarada S, et al: Assessment of culprit lesion morphology in acute myocardial infarction: ability of optical coherence tomography compared with intravascular ultrasound and coronary angioscopy. J Am Coll Cardiol 2007; 50: 933-939

26) Tearney GJ, Regar E, Akasaka T, et al: Consensus standards for acquisition, measurement, and reporting of intravascular optical coherence tomography studies: a report from the International Working Group for Intravascular Optical Coherence Tomography Standardization and Validation. J Am Coll Cardiol 2012; 59: $1058-1072$

27) Soeda $T$, Uemura S, Morikawa $Y$, et al: Diagnostic accuracy of dual-source computed tomography in the characterization of coronary atherosclerotic plaques: comparison with intravascular optical coherence tomography. Int J Cardiol 2011; 148: 313-318

28) Vergallo R, Yonetsu T, Kato K, et al: Evaluation of culprit lesions by optical coherence tomography in patients with st-elevation myocardial infarction. Int J Cardiol 2013; 168: 15921593

29) Falk E: Pathogenesis of atherosclerosis. J Am Coll Cardiol 2006; 46: C7-C12

30) Schaar JA, Muller JE, Falk E, et al: Terminology for high-risk and vulnerable coronary artery plaques. Report of a meeting on the vulnerable plaque, June 17 and 18, 2003, Santorini, Greece. Eur Heart J 2004; 25: 1077-1082

31) Narula J, Garg P, Achenbach S, et al: Arithmetic of vulnerable plaques for noninvasive imaging. Nat Clin Pract Cardiovasc Med 2008; 5(Suppl 2): S2-S10

32) Virmani R, Burke AP, Farb A, et al: Pathology of the vulnerable plaque. J Am Coll Cardiol 2006; 47: C13- C18

33) Doyle B, Caplice N: Plaque neovascularization and antiangiogenic therapy for atherosclerosis. J Am Coll Cardiol 2007; 49: 2073-2080

34) Burke AP, Farb A, Malcom GT, et al: Coronary risk factors and plaque morphology in men with coronary disease who died suddenly. N Engl J Med 1997; 336: 1276-1282

35) Virmani R, Kolodgie FD, Burke AP, et al: Lessons from sudden coronary death: a comprehensive morphological classification scheme for atherosclerotic lesions. Arterioscler Thromb Vasc Biol 2000; 20: 1262-1275

36) Kolodgie FD, Gold HK, Burke AP, et al: Intraplaque hemorrhage and progression of coronary atheroma. N Engl J Med 2003; 349: 2316-2325

37) Takano M, Jang IK, Inami S, et al: In vivo comparison of optical coherence tomography and angioscopy for the evaluation of coronary plaque characteristics. Am J Cardiol 2008; 101: 471-476

38) Kubo T, Imanishi $T$, Takarada $S$, et al: Implication of plaque color classification for assessing plaque vulnerability: a coronary angioscopy and optical coherence tomography investigation. JACC Cardiovasc Interv 2008; 1: 74-80 
39) Raffel OC, Merchant FM, Tearney GJ, et al: In vivo association between positive coronary artery remodelling and coronary plaque characteristics assessed by intravascular optical coherence tomography. Eur Heart J 2008; 29: 1721-1728

40) Bouki KP, Katsafados MG, Chatzopoulos DN, et al: Inflammatory markers and plaque morphology: an optical coherence tomography study. Int J Cardiol 2012; 154: 287-292

41) Koga S, Ikeda S, Yoshida T, et al: Elevated levels of systemic pentraxin 3 are associated with thin-cap fibroatheroma in coronary culprit lesions: assessment by optical coherence tomography and intravascular ultrasound. JACC Cardiovasc Interv 2013; 6: 945-954

42) Fleiner M, Kummer M, Mirlacher M, et al: Arterial neovascularization and inflammation in vulnerable patients: early and late signs of symptomatic atherosclerosis. Circulation 2004; 110: 2843-2850

43) MacNeill BD, Jang IK, Bouma BE, et al: Focal and multi-focal plaque macrophage distributions in patients with acute and stable presentations of coronary artery disease. J Am Coll Cardiol 2004; 44: 972-979

44) Tearney GJ, Yabushita H, Houser SL, et al: Quantification of macrophage content in atherosclerotic plaques by optical coherence tomography. Circulation 2003; 107: 113-119

45) Raffel OC, Tearney GJ, Gauthier DD, et al: Relationship between a systemic inflammatory marker, plaque inflammation, and plaque characteristics determined by intravascular optical coherence tomography. Arterioscler Thromb Vasc Biol 2007; 27: 1820-1827

46) Kitabata H, Tanaka A, Kubo T, et al: Relation of microchannel structure identified by optical coherence tomography to plaque vulnerability in patients with coronary artery disease. Am J Cardiol 2010; 105: 1673-1678

47) Tian J, Hou J, Xing L, et al: Significance of intraplaque neovascularisation for vulnerability: optical coherence tomography study. Heart 2012; 98: 1504-1509

48) Uemura $S$, Ishigami $K$, Soeda $T$, et al: Thin-cap fibroatheroma and microchannel findings in optical coherence tomography correlate with subsequent progression of coronary atheromatous plaques. Eur Heart J 2012; 33: 78-85

49) Davies MJ, Thomas A: Thrombosis and acute coronary-artery lesions in sudden cardiac ischemic death. N Engl J Med 1984; 310: $1137-1140$

50) Grønholdt ML, Dalager-Pedersen S, Falk E: Coronary atherosclerosis: determinants of plaque rupture. Eur Heart J 1998; 19(Suppl C): C24-C29

51) McGill HC, McMahan CA, Malcom GT, et al: Relation of glycohemoglobin and adiposity to atherosclerosis in youth. Pathobiological Determinants of Atherosclerosis in Youth (PDAY) Research Group. Arterioscler Thromb Vasc Biol 1995; 15: 431-440

52) Finn AV, Nakano M, Narula J, et al: Concept of vulnerable/unstable plaque. Arterioscler Thromb Vasc Biol 2010; 30: 1282-1292

53) Yonetsu T, Kakuta T, Lee T, et al: In vivo critical fibrous cap thickness for rupture-prone coronary plaques assessed by optical coherence tomography. Eur Heart J 2011; 32: 1251-1259
54) Tanaka A, Imanishi T, Kitabata H, et al: Morphology of exertion-triggered plaque rupture in patients with acute coronary syndrome: an optical coherence tomography study. Circulation 2008; 118: 2368-2373

55) Davies MJ, Bland JM, Hangartner JR, et al: Factors influencing the presence or absence of acute coronary artery thrombi in sudden ischaemic death. Eur Heart J 1989; 10: 203-208

56) Burke AP, Kolodgie FD, Farb A, et al: Healed plaque ruptures and sudden coronary death: evidence that subclinical rupture has a role in plaque progression. Circulation 2001; 103: 934-940

57) Kato K, Yonetsu T, Kim SJ, et al: Nonculprit plaques in patients with acute coronary syndromes have more vulnerable features compared with those with non-acute coronary syndromes: a 3 -vessel optical coherence tomography study. Circ Cardiovasc Imaging 2012; 5: 433-440

58) Kato K, Yonetsu T, Jia H, et al: Nonculprit coronary plaque characteristics of chronic kidney disease. Circ Cardiovasc Imaging 2013; 6: 448-456

59) Mann J, Davies MJ: Mechanisms of progression in native coronary artery disease: role of healed plaque disruption. Heart 1999; 82: 265-268

60) Arbab-Zadeh A, Nakano M, Virmani R, et al: Acute coronary events. Circulation 2012; 125: 1147-1156

61) Lee JB, Mintz GS, Lisauskas JB, et al: Histopathologic validation of the intravascular ultrasound diagnosis of calcified coronary artery nodules. Am J Cardiol 2011; 108: 1547-1551

62) Jia H, Abtahian F, Aguirre AD, et al: In vivo diagnosis of plaque erosion and calcified nodule in patients with acute coronary syndrome by intravascular optical coherence tomography. J Am Coll Cardiol 2013; 62: 1748-1758

63) Mortensen KH, Thuesen L, Kristensen IB, et al: Spontaneous coronary artery dissection: a Western Denmark Heart Registry study. Catheter Cardiovasc Interv 2009; 74: 710-717

64) Vrints CJ: Spontaneous coronary artery dissection. Heart 2010; 96: 801-808

65) Alfonso F, Canales E, Aleong G: Spontaneous coronary artery dissection: diagnosis by optical coherence tomography. Eur Heart J 2009; 30: 385

66) Poon K, Bell B, Raffel OC, et al: Spontaneous coronary artery dissection: utility of intravascular ultrasound and optical coherence tomography during percutaneous coronary intervention. Circ Cardiovasc Interv 2011; 4: e5-e7

67) Alfonso F, Paulo M, Gonzalo N, et al: Diagnosis of spontaneous coronary artery dissection by optical coherence tomography. J Am Coll Cardiol 2012; 59: 1073-1079

68) Morikawa $\mathrm{Y}$, Uemura S, Ishigami K, et al: Morphological features of coronary arteries in patients with coronary spastic angina: assessment with intracoronary optical coherence tomography. Int J Cardiol 2011; 146: 334-340

69) Tanaka A, Shimada K, Tearney GJ, et al: Conformational change in coronary artery structure assessed by optical coherence tomography in patients with vasospastic angina. J Am Coll Cardiol 2011; 58: 1608-1613 